\title{
Incidence, risk factors, clinical characteristics and outcomes of deep venous thrombosis in patients with COVID-19 attending the Emergency Department: results of the UMC-19-S
}

Sònia Jiménez $z^{\mathrm{a} \star}$, Óscar Mirón ${ }^{\mathrm{a} *}$, Pere Llorens ${ }^{\mathrm{b}}$, Francisco J. Martín-Sánchez ${ }^{c}$, Guillermo Burillo-Putze ${ }^{d}$, Pascual Piñera ${ }^{e}$, Alfonso Martín ${ }^{\dagger}$, Aitor Alquézar ${ }^{\mathrm{g}}$, Eric J. Garcia-Lamberechts ${ }^{\mathrm{c}}$, Javier Jacob ${ }^{\mathrm{h}}$, María L. L. Grimai, Javier Millánj, Francisca Molinak ${ }^{k}$, Patricia B. Albero', Carlos Cardozo ${ }^{\mathrm{a}, \mathrm{m}}$, Josep M. Mòdol ${ }^{\mathrm{n}}$, Alfons Aguirre ${ }^{\circ}$, Ruth Gaya ${ }^{\mathrm{p}}$, Maria Adroher ${ }^{\mathrm{q}}$, Lluis Llauger', Juan J. L. Díaz', Nayra C. Gonzálezt, Paula L. Aragües ${ }^{e}$, Ana P. Gómez $z^{u}$ and Juan G. del Castillo ${ }^{\circ}$; on behalf of the Spanish Investigators on Emergency Situations TeAm (SIESTA) network*

\begin{abstract}
Background and importance A higher incidence of venous thromboembolism [both pulmonary embolism and deep vein thrombosis (DVT)] in patients with coronavirus disease 2019 (COVID-19) has been described. But little is known about the true frequency of DVT in patients who attend emergency department (ED) and are diagnosed with COVID-19.
\end{abstract}

Objective We investigated the incidence, risk factors, clinical characteristics and outcomes of DVT in patients with COVID-19 attending the ED before hospitalization.

Methods We retrospectively reviewed all COVID patients diagnosed with DVT in 62 Spanish EDs (20\% of Spanish EDs, case group) during the first 2 months of the COVID-19 outbreak. We compared DVT-COVID-19 patients with COVID-19 without DVT patients (control group). Relative frequencies of DVT were estimated in COVID and non-COVID patients visiting the ED and annual standardized incidences were estimated for both populations. Sixty-three patient characteristics and four outcomes were compared between cases and controls.

Results We identified 112 DVT in 74814 patients with COVID-19 attending the ED [1.50\%; 95\% confidence interval $(\mathrm{Cl}), 1.23-1.80 \%$ ]. This relative frequency was similar than that observed in non-COVID patients [2109/1 388879; $1.52 \% 0 ; 95 \% \mathrm{Cl}, 1.45-1.69 \%$; odds ratio $(O R)=0.98$ [0.82-1.19]. Standardized incidence of DVT was higher in COVID patients (98,38 versus 42,93/100,000/ year; OR, 2.20; 95\% Cl, 2.03-2.38). In COVID patients, the clinical characteristics associated with a higher risk of presenting DVT were older age and having a history of venous thromboembolism, recent surgery/immobilization and hypertension; chest pain and desaturation at ED arrival and some analytical disturbances were also more frequently seen, $\mathrm{D}$-dimer $>5000 \mathrm{ng} / \mathrm{mL}$ being the strongest. After adjustment for age and sex, hospitalization, ICU admission and prolonged hospitalization were more frequent in cases than controls, whereas mortality was similar (OR, 1.37; 95\% Cl, 0.77-2.45).

Conclusions DVT was an unusual form of COVID presentation in COVID patients but was associated with a worse prognosis. European Journal of Emergency Medicine 28: 218-226 Copyright (C) 2021 Wolters Kluwer Health, Inc. All rights reserved.

European Journal of Emergency Medicine 2021, 28:218-226

Keywords: clinical characteristics, coronavirus disease $\mathbf{2 0 1 9}$, deep venous thrombosis, incidence, outcome, risk factors, severe acute respiratory syndrome coronavirus 2

${ }^{a}$ Emergency Department, Hospital Clínic, IDIBAPS, University of Barcelona, Barcelona, Catalonia, ${ }^{b}$ Emergency Department, Hospital General de Alicante, University Miguel Hernández, Elche, Alicante, ${ }^{\circ}$ Emergency Department, Hospital Clínico San Carlos, IDISSC, Universidad Complutense, Madrid, ${ }^{d}$ Emergency Department, Hospital Universitario de Canarias, Tenerife, ${ }^{e}$ Emergency Department, Hospital General Universitario Reina Sofia, Murcia, 'Servicio de Urgencias, Hospital Universitario de Móstoles,

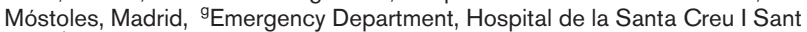
Pau, hEmergency Department, Hospital Universitari de Bellvitge, L'Hospitalet de Llobregat, Barcelona, Catalonia, 'Emergency Department, Hospital Universitario Doctor Peset Aleixandre, 'Emergency Department, Hospital Universitario y Politécnico La Fe, Valencia, ${ }^{k}$ Emergency Department, Hospital Universitario General de Alicante, 'Emergency Department, Hospital Virgen de los Lirios, Alcoy, Alicante, Spain ${ }^{m}$ Emergency Department, Hospital Austral, Buenos Aires, Argentina, "Emergency Department, Hospital Universitari Germans Trias i Pujol, Badalona, ${ }^{\circ}$ Emergency Department, Hospital del Mar, Barcelona, PEmergency Department, Hospital Universitari Joan XXIII, Tarragona, ${ }^{\mathrm{q} E m e r g e n c y ~ D e p a r t m e n t, ~ H o s p i t a l ~ U n i v e r s i t a r i ~ d e ~ G i r o n a ~ D r . ~ J o s e p ~}$ Trueta, Girona, 'Emergency Department, Hospital Universitari de Vic, Barcelona Catalonia, 'smergency Department, Hospital Universitario Lucus Augusti, Lugo, 'Emergency Department, Hospital Universitario de Gran Canaria Dr. Negrín, Las Palmas de Gran Canaria and "Emergency Department, Hospital de Lliria, Valencia, Spain

Correspondence to Sònia Jiménez, MD, PhD, Emergency Department, Hospital Clínic, Villarroel 170, 08036 Barcelona, Catalonia, Spain

Tel: +349322798 33; fax: +349322756 93; e-mail: sjimenez@clinic.cat

${ }^{*}$ Dr. Sònia Jiménez and Dr. Òscar Miró equally contributed to the writing of this article.

**The SIESTA network is formed by the following researchers and centers (all from Spain)

Received 27 August 2020 Accepted 24 November 2020 


\section{Introduction}

Infection by severe acute respiratory syndrome coronavirus 2 (SARS-CoV-2) is mainly characterized by fever and respiratory symptoms, with dyspnea and lung infiltrates being present in more than $50 \%$ of hospitalized cases [1]. A significant number of other signs and symptoms can be present, involving the gastrointestinal tract, hepatic inflammation, myalgia and rhabdomyolysis [1-3]. The association of systemic coagulation activation is particularly frequent in coronavirus disease 2019 (COVID-19), especially in severe cases [1-5]. The hemostatic abnormality most consistently observed in COVID-19 is increased D-dimers in the blood stream, which is associated with a higher risk of requiring mechanical ventilation, ICU admission and death [6]. The character of this coagulopathy is quite prothrombotic with a high risk of venous thromboembolism (VTE) [7,8]. Several studies have shown an increased incidence of VTE, involving both pulmonary embolism and deep vein thrombosis (DVT) in patients admitted to ICUs, with cumulative incidences of up to $35 \%$ [9-12]. In general ward hospitalization, a recent study [11] described an incidence of asymptomatic DV'T in COVID patients similar to that reported in other studies on asymptomatic DV'T in nonCOVID patients [13]. The largest study included 338 COVID patients, 61 of whom were admitted to an ICU and reported thromboembolic events in $7.7 \%$ of cases, with a cumulative rate of $21 \%$ [4]. The authors noted that half of the V'TE events were diagnosed after $24 \mathrm{~h}$ of hospital admission. In this scenario, it is difficult to quantify the real association of DV'T with the pathogenesis of the disease caused by SARS-CoV-2 infection, because the appearance of DV'T may be favored by the fact that hospitalized patients are usually bedridden, multidrug-treated and in very poor condition. In addition, irregular initiation of thromboprophylaxis during the COVID-19 pandemic makes it more difficult to interpret the real risk of DVT associated with COVID-19 itself.

Bearing this uncertainty in mind, in the present study, we aimed to investigate the incidence of DVT in patients attending the emergency department (ED) before hospitalization and treatment with anticoagulants and specific drugs for SARS-CoV-2 infection. The specific objectives were: (1) to estimate the relative frequency and annual standardized incidence of DV'T in patients with COVID19; (2) to uncover the risk factors associated with the development of DV'T in patients with COVID-19 and (3) to investigate the outcomes of COVID patients presenting DVT.

\section{Methods}

\section{Study design and setting}

This was a retrospective, case-control, ED-based, multicenter study that reviewed the medical reports of COVID-19 patients attended at Spanish EDs who were diagnosed as having objective DVT during ED assessment and management, before hospitalization (cases). In Spain, the first case of SARS-CoV-2 infection was detected on 31 January 2020 and, accordingly, the definition of the COVID-19 period for patient inclusion in the present study was set from 1 March 2020 to 30 April 2020. During this 61-day period, 213435 cases of COVID-19 were confirmed by the Spanish Ministry of Health [14].

The present study forms part of the Unusual Manifestations of COVID-19 (UMC-19) project, which was designed to investigate the potential relationship between COVID-19 and 10 different entities that could be influenced by SARS-CoV-2 infection itself and for which at least one case had been published, suggesting a potential link with this viral infection. The main objectives of the UMC-19 project were common for all 10 entities, and consisted in the description of the incidence, risk factors, clinical characteristics and outcomes for each particular entity (cases).

The investigators of the UMC-19 project initially contacted 152 Spanish EDs, which roughly constitute half of the 312 hospital EDs of the Spanish public health network. Of these, 81 were interested in participating and analyzed the protocol, and 62 finally consented to participate and duly sent all the data required (Fig. 1). These 62 hospitals provide a quite balanced representation of the Spanish territory (12 of the 17 Spanish autonomous communities were represented), type of hospitals (community, reference and high-technology university hospitals were included) and involvement in the pandemic (with EDs attending from 1-47\% of ED census corresponding to COVID patients during the COVID outbreak period). These EDs have a reference population of 15.5 million people, which constitutes about $33 \%$ of the whole Spanish population (46.9 million) $[15,16]$.

The present study was formed by two different parts to cover the objectives. The first part, covering specific objective 1, was related with the estimation of relative frequency and annual standardized incidence of DVT in ED comers and population and comparisons with figures in non-COVID comers and population. With this purpose, we collected the ED census in March/April 2020 (COVID period) and distinguish between COVID and non-COVID patients, as well as the ED census in March/ April 2019 (pre-COVID period), all corresponding to nonCOVID patients, in order to take into account potential differences in ED comers due to pandemic lockdown. The number of DVT diagnosis performed at the ED at each period and patients' group was recorded.

The second part, covering specific objectives 2 and 3, compared risk factors, clinical characteristics and outcomes in COVID patients with and without DVT. The diagnosis of COVID-19 was based on SARS-Cov-2 antigen detection in a nasopharyngeal swab by reverse 


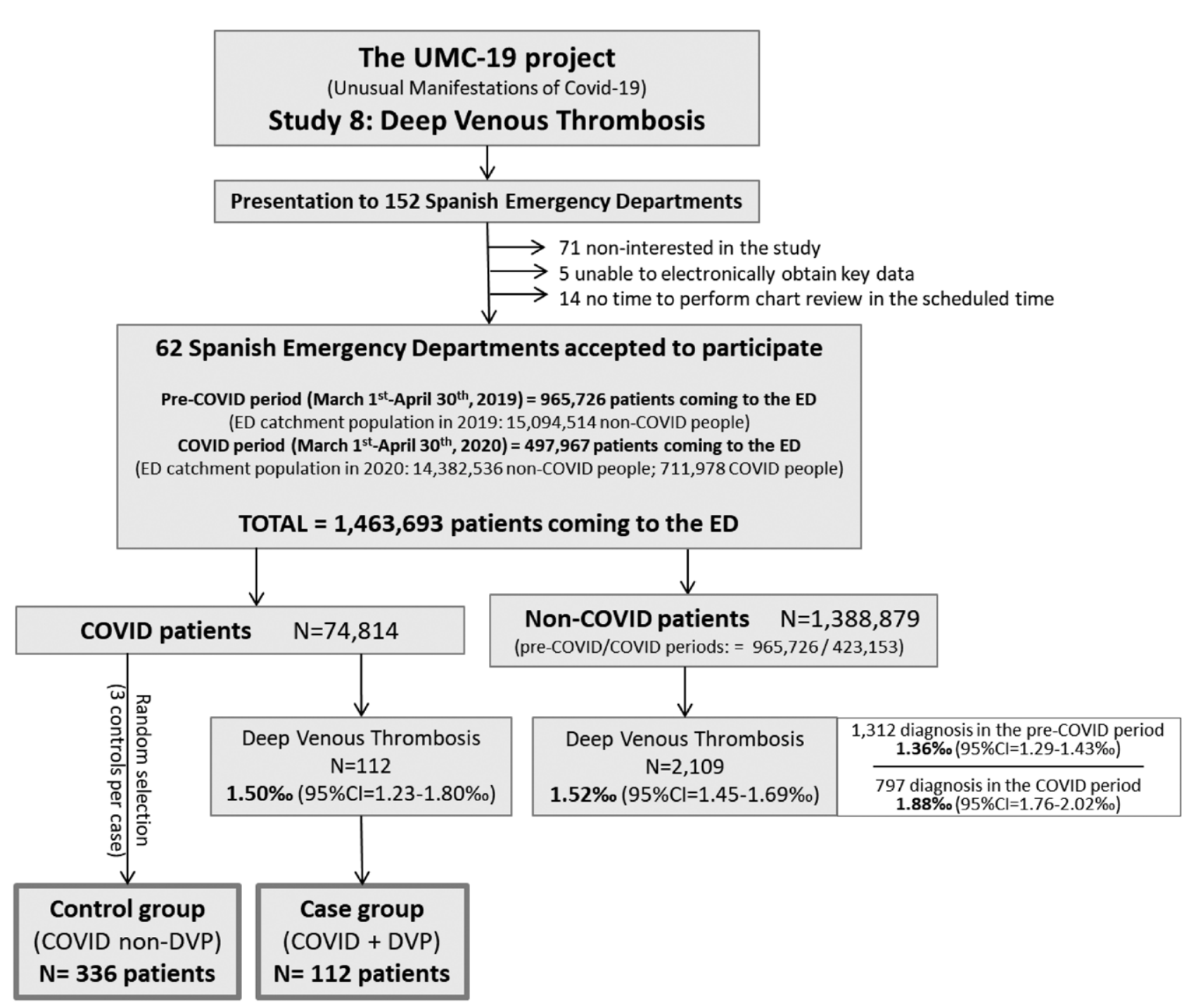

Study design and inclusion flow chart.

transcriptase PCR (RT-PCR), a clinically compatible clinical picture (including at least malaise, fever and cough) or the presence of the typical lung parenchymal infiltrates in chest X-ray or pulmonary computerized tomography (CT) in patients with some clinical symptoms attributable to COVID-19. The case group (COVID patients with DVT) was formed by patients diagnosed with DVT during the ED stay. The diagnosis was based on complete compression Doppler ultrasound (CCUS) of the legs, which included the proximal and distal territory, performed by a radiologist. The control group (COVID patients without DVT) was formed by selecting three COVID patients without DVT for every case detected by each center. Selection was randomly performed by the inclusion of the three COVID patients seen immediately before or after each case included by the center. This group was specifically designed to uncover the risk factors for DVT development in COVID patients.

\section{Independent variables}

We collected 63 independent variables, which included 2 demographic data (age and sex), 6 risk factors for DVT (estrogen use, previous history of DVT, surgery/ immobilization previous month, active cancer, obesity and smoking habit), 10 comorbidities (hypertension, dyslipidemia, diabetes mellitus, coronary artery disease, cerebrovascular disease, chronic obstructive pulmonary disease, chronic liver disease, asthma, chronic kidney disease - creatinine $>2 \mathrm{mg} / \mathrm{dL}$ - and dementia), 17 symptoms recorded at ED arrival (time elapsed from symptom 
onset to ED attendance, fever, rhinorrhea, cough, expectoration, dyspnea, chest pain, syncope, hemoptysis, leg pain/edema, abdominal pain, vomiting, diarrhea, confusion, headache, anosmia and dysgeusia), 5 vital signs at ED arrival (temperature, systolic blood pressure, heart rate, respiratory rate and room air pulsioxymetry), 15 laboratory parameters [C-reactive protein, creatinine, aspartate aminotransferase, bilirubin, lactate dehydrogenase (LDH), procalcitonin, hemoglobin, leucocyte, lymphocyte and platelet counts, activated partial thromboplastin time, prothrombin time, fibrinogen, and D-dimer], 4 radiological findings in chest X-ray (cardiomegaly, lung interstitial infiltrates, ground-glass opacities and patchy shadowing, and pleural effusion), 3 findings in pulmonary CT (pulmonary embolism, lung interstitial infiltrates and lung ground-glass parenchymal opacities) and location of DVT in CCUS (proximal, distal or both).

\section{Outcomes}

We defined four different outcomes for cases and controls, which consisted of: (1) the need for hospitalization; (2) the need for ICU admission; (3) prolonged hospitalization (considered if length of hospitalization from ED arrival to discharge was longer than 7 days, which is the median length of hospitalization in Spain) and (4) in-hospital all-cause mortality.

\section{Statistical analysis}

Discrete variables were expressed as absolute values and percentages, and continuous variables median and interquartile range if not normally distributed. Relative frequency of DVT was expressed per thousand (\%o) of COVID or non-COVID patients coming to the ED, and annual standardized incidence was expressed per 100000 COVID or non-COVID individuals. Both estimations were made with $95 \%$ confidence intervals (CI) that were calculated using the exact method for binomial distributions. To estimate COVID and non-COVID population in each ED catchment area, we used the seroprevalence of SARS-CoV-2 in the province where the ED is placed. These detailed seroprevalences have been determined in a wide Spanish study performed between 27 April 2020 and 11 May 2020 and have been recently reported [17].

Differences between case and control groups were assessed by the chi-square test (or Fisher's exact test if needed) for qualitative variables, and the Mann-Whitney nonparametric for quantitative variables. The magnitude of associations remaining statistically significant after such correction was expressed as the unadjusted odds ratio (OR) with 95\% CI, that were calculated by logistic regression independently comparing all cases with all control A patients. The magnitude of the associations was expressed as unadjusted OR with 95\% CI, and for these estimations, the continuous variables were dichotomized using clinically meaningful cutoffs or values around the median. As the number of cases expected to be identified was not large, we did not plan to go further in the investigation of the significant relationships identified in the unadjusted analysis by using adjusted models. The only exception was outcomes, which were adjusted for age and sex. In all comparisons, statistical significance was accepted if the $P$ value was $<0.05$ or if $95 \%$ CI of the risk estimations excluded value 1 . The analyses were performed with the SPSS (v.24) statistical software package (IBM, Armonk, New York, USA).

\section{Ethics}

The UMC-19 project was approved by the ethics committee of the Hospital Clínic of Barcelona (Spain), with the reference number $\mathrm{HCB} / 2020 / 0534$, that acted as the central ethical committee. Under the exceptional circumstances generated by the COVID-19, and taking into account the urgent need to obtain feasible data related to this new disease, and the noninterventional and retrospective nature of the project, the need to obtain written permission from patients to be included in the study was waived. All patients were codified by investigators of the participating centers before entering their data into the general database, and thus, patient identity remained anonymous for investigators who analyzed the database. The UMC-19-S 8 was carried out in strict compliance with the Declaration of Helsinki principles. The authors designed the study, gathered and analyzed the data, vouched for the data and analysis, wrote the article and decided to publish.

\section{Results}

\section{Relative frequency and standardized incidence}

A total of 74814 (15\%o) patients with COVID-19 were seen in the 61 Spanish EDs participating in the UMC$19-S_{8}$ (Fig. 1) during the 61-day study period. We identified 112 DVT in COVID-19 patients and constituted the case group. The control group was formed by 336 randomly selected COVID patients without DVT (COVID-19 non-DVT) during the same period based on a case-control ratio of 3:1. Confirmation of COVID-19 infection by RT-PCR was performed in 71.4 and $73.2 \%$ of cases and controls, respectively. In total $28.6 \%$ cases and $26.8 \%$ controls were diagnosed by a clinically compatible clinical picture (including at least malaise, fever and cough) or the presence of the typical lung parenchymal infiltrates in chest X-ray or pulmonary CT in patients with some clinical symptoms attributable to COVID-19.

Totally 1388879 non-COVID-19 patients were seen during the 122-day period (497967 during the 61 days in the 2020 COVID-19 period and 965726 during the 61 days in the 2019 pre-COVID-19 period). Of them, 2109 were diagnosed with DVT (797 during COVID-19 period and 1312 during pre-COVID-19 period). Accordingly, relative frequency for DVT in COVID-19 patients was 
Table 1 Baseline characteristics of patients with COVID-19 with deep venous thrombosis (cases) and comparison with patients with COVID-19 without deep venous thrombosis (control group)

\begin{tabular}{|c|c|c|c|}
\hline & $\begin{array}{c}\text { Cases (COVID } \\
\text { with DVT) } \\
N=112 n(\%)\end{array}$ & $\begin{array}{c}\text { Control group } \\
\text { (COVID without } \\
\text { DVT) } N=336 \\
n(\%)\end{array}$ & $P$ value \\
\hline \multicolumn{4}{|l|}{ Demographics } \\
\hline Age (years) & $68(59-78)$ & $65(52-77)$ & 0.07 \\
\hline Age $>65$ & $69(61.6)$ & $169(50.3)$ & 0.03 \\
\hline Sex (female) & $44(60.7)$ & $160(47.6)$ & 0.12 \\
\hline \multicolumn{4}{|l|}{ Risk factors for VTE } \\
\hline Obesity (clinically estimated) & $23(20.5)$ & $51(15.2)$ & 0.18 \\
\hline Active cancer & $17(15.2)$ & $32(9.5)$ & 0.09 \\
\hline Surgery/immobilization $<1$ month & $14(12.5)$ & $14(4.2)$ & 0.002 \\
\hline History of VTE & $12(10.7)$ & $13(3.9)$ & 0.006 \\
\hline Active smoker & $8(7.1)$ & $22(6.5)$ & 0.86 \\
\hline Estrogen use & $1(0.9)$ & $4(1.2)$ & 0.79 \\
\hline \multicolumn{4}{|l|}{ Other comorbidities } \\
\hline Hypertension & $66(58.9)$ & $152(45.2)$ & 0.01 \\
\hline Dyslipidemia & $34(30.4)$ & 112 (33.3) & 0.56 \\
\hline Diabetes mellitus & $19(17)$ & $60(17.9)$ & 0.83 \\
\hline Chronic kidney disease & $8(7.1)$ & $21(6.3)$ & 0.73 \\
\hline Coronary artery disease & $7(6.3)$ & $26(7.7)$ & 0.60 \\
\hline Cerebrovascular disease & $7(6.3)$ & $23(6.8)$ & 0.82 \\
\hline Dementia & $6(5.4)$ & $29(8.6)$ & 0.26 \\
\hline $\begin{array}{l}\text { Chronic obstructive pulmonary } \\
\text { disease }\end{array}$ & $6(5.4)$ & $29(8.6)$ & 0.26 \\
\hline Chronic liver disease & $5(4.5)$ & $12(3.6)$ & 0.66 \\
\hline Asthma & $5(4.5)$ & $24(7.1)$ & 0.31 \\
\hline
\end{tabular}

Bold $P$ values denote statistical significance $(P<0.05)$.

COVID, coronavirus disease; DVT, deep venous thrombosis; VTE, venous thromboembolism.

$1.50 \%$ ( $95 \%$ CI, $1.23-1.80$ ) and relative frequency for DVT in non-COVID-19 patients was $1.52 \%$ o (95\% CI, $1.45-1.69)$ [with relative frequencies of $1.36 \%$ (95\% CI, $1.29-1.43$ ) and $1.88 \%$ (95\% CI, 1.76-2.02) when COVID19 and pre-COVID-19 periods were analyzed individually] (Fig. 1). Therefore, relative frequency of DVT in COVID-19 patients compared to non-COVID patients coming to the ED resulted in an unadjusted OR of 0.98 (95\% CI, 0.82-1.19) [1.10 (95\% CI, 0.91-1.34) and 0.79 (95\% CI, 0.65-0.97) when comparisons were made with non-COVID-19 patients diagnosed during the preCOVID-19 and COVID-19 periods separately].

On the other hand, the annual standardized incidences of DVT were 98,38 per 100000 COVID individuals and year and 42,93 per 100000 non-COVID individuals per year (with partial standardized incidences of 33,25 and 52,15 for the COVID and pre-COVID periods considered individually). Accordingly, the OR for standardized incidence in COVID patients with respect to non-COVID patients was 2.20 (95\% CI, 2.03-2.38) [2.80 (95\% CI, 2.62-3.08) and 1.81 (95\% CI, 1.67-1.96) for the COVID and preCOVID periods, respectively].

\section{Clinical characteristics}

The mean age of COVID patients with DVT (cases) was 67 years: $61 \%$ were males, $11 \%$ had VTE history and $15 \%$ have active cancer. Other common comorbidities were hypertension (59\%) and dyslipidemia (30\%) (Table 1). The most frequent symptomatology was dyspnea (59\%), lower limb pain/edema (58\%), fever (41.1\%), cough (36.6\%) and chest pain (20.7\%), and the median time from the symptom onset to ED consultation was 7 days. Table 2 shows the remaining clinical characteristics, as well as the vitals at ED arrival and laboratory findings. The median D-dimer levels in these patients were $6.021 \mathrm{ng} / \mathrm{mL}$ and 68 patients $(72.3 \%)$ had D-dimer levels above $3000 \mathrm{ng} / \mathrm{mL}$ (Table 2). The location of DVT in complete compression Doppler ultrasound was only proximal in $40 \%$, only distal in $38 \%$ and proximal and distal in $22 \%$ of cases.

Some statistically significant differences were found when cases were compared with controls (Tables 1-2), and the magnitude of these associations is shown in Table 3. The baseline characteristics in COVID patients associated with a higher risk of presenting DVT were age older than 65 years (OR, 1.50; 95\% CI, 1.02-2.45) and having a history of VTE $(2.98$; 95\% CI, 1.31-6.76), surgery or immobilization in the past month (OR, 3.28; 95\% CI, 1.51-7.12) and hypertension (OR, 1.73; 95\% CI, 1.12-2.67). Chest pain (OR, 1.8; 95\% CI, 1.03-3.17) and desaturation (OR, 1.99; 95\% CI, 1.23-3.21) at ED arrival, and some analytical disturbances, such as LDH values above $350 \mathrm{IU} / \mathrm{L}$ (OR, 2.0; 95\% CI, 1.22-3.27) and leucocyte count above $10 \times 10^{3}$ cells $/ \mu \mathrm{L}(\mathrm{OR}, 4.65 ; 95 \% \mathrm{CI}$, 2.91-7.43), were associated with a higher risk of presenting DVT in COVID patients. Of note, D-dimer levels greater than $5000 \mathrm{ng} / \mathrm{mL}$ were strongly associated with the presence of DVT (OR, 19.44; 95\% CI, 10.53-35.88).

\section{Outcomes}

Nearly all the outcomes measured were worse in the cases than in the control group (Fig. 2). Specifically, COVID patients with DVT had a greater risk of the need for hospitalization (OR, 2.96; 95\% CI, 1.51-5.79), need for ICU admission (OR, 3.67; 95\% CI, 1.93-6.97) and prolonged hospitalization (OR, 3.01; 95\% CI, 1.88-4.82) compared to COVID patients without DVT, but the in-hospital mortality was similar. The sensitivity analysis including only COVID patients microbiologically confirmed by RT-PCR ( 80 cases and 246 controls) did not show differences in outcomes.

\section{Discussion}

The results of UMC-19-S show that around $1.50 \%$ of COVID-19 patients in EDs had DVT, being a relative frequency that is similar to what was observed in patients without COVID (1.52\%o). However, a lower relative frequency of DVT was recorded in non-COVID patients during the COVID period (OR, 0.79). Furthermore, the annual standardized incidence was also lower in non-COVID patients during the COVID period. These data could indicate that patients without COVID consulted less in the EDs, and also that the active search for DVT in these patients was greater. This picture is supported by the fact that more than a third did not present 
Table 2 Clinical and analytical characteristics of patients with COVID-19 with deep venous thrombosis (cases) and comparison with patients with COVID-19 without deep venous thrombosis (control group)

\begin{tabular}{|c|c|c|c|}
\hline & $\begin{array}{l}\text { Cases (COVID with } \\
\text { DVT) } N=112 n(\%)\end{array}$ & $\begin{array}{c}\text { Control group (COVID } \\
\text { without DVT) } N=336 n(\%)\end{array}$ & $P$ value \\
\hline \multicolumn{4}{|l|}{ Symptoms at ED arrival } \\
\hline Lasting symptoms (days) [median (IQR)] & $7(3-14)$ & $7(3-10)$ & 0.39 \\
\hline Dyspnea & $66(58.9)$ & $187(55.7)$ & 0.54 \\
\hline Lower limb pain/edema & $65(58)$ & - & - \\
\hline Fever & $46(41.1)$ & $198(58.9)$ & 0.001 \\
\hline Cough & $41(36.6)$ & $197(58.6)$ & $<0.001$ \\
\hline Chest pain & $23(20.5)$ & $42(12.5)$ & 0.03 \\
\hline Diarrhea & $17(15.2)$ & $55(16.4)$ & 0.76 \\
\hline Expectoration & $14(12.5)$ & $49(14.6)$ & 0.58 \\
\hline Confusion & $9(8)$ & $23(6.8)$ & 0.67 \\
\hline Dysgeusia & $9(8)$ & $26(7.7)$ & 0.91 \\
\hline Anosmia & $7(6.3)$ & $22(6.5)$ & 0.91 \\
\hline Abdominal pain & $6(5.4)$ & $17(5.1)$ & 0.90 \\
\hline Vomiting & $6(5.4)$ & $25(7.4)$ & 0.45 \\
\hline Headache & $6(5.4)$ & $39(11.6)$ & 0.05 \\
\hline Syncope & $3(2.7)$ & $14(4.2)$ & 0.47 \\
\hline Rhinorrhea & $2(1.8)$ & $23(6.8)$ & 0.04 \\
\hline Hemoptysis & $2(1.8)$ & $2(0.6)$ & 0.24 \\
\hline \multicolumn{4}{|l|}{ Signs at ED arrival } \\
\hline Temperature $\left({ }^{\circ} \mathrm{C}\right)$ [median (IOR)] & $36.5(36.0-37.2)$ & $36.6(36.0-37.3)$ & 0.45 \\
\hline Temperature at $\mathrm{ED}$ arrival $>38^{\circ} \mathrm{C}$ & $10(9)$ & $35(10.6)$ & 0.62 \\
\hline $\mathrm{SBP}(\mathrm{mmHg})[$ median (IQR)] & $131(116-144)$ & $125(115-140)$ & 0.36 \\
\hline Heart rate $(\mathrm{bpm})[$ median $(\mathrm{IQR})]$ & $92(85-107)$ & $88(78-100)$ & 0.002 \\
\hline Heart rate $>100 \mathrm{bpm}$ & $38(33.9)$ & $86(26.1)$ & 0.11 \\
\hline Respiratory rate (bpm) [median (IQR)] & $20(16-24)$ & $18(16-22)$ & 0.07 \\
\hline Respiratory rate $>20 \mathrm{bpm}$ & $58(53.2)$ & $146(45.2)$ & 0.14 \\
\hline Room air pulsioxymetry (\%) [median (IQR)] & $95(91-97)$ & $96(93-98)$ & 0.003 \\
\hline Room air pulsioxymetry $<96 \%$ & $84(75)$ & $202(61)$ & 0.008 \\
\hline \multicolumn{4}{|l|}{ Laboratory findings ${ }^{a}$ ( $n$ of cases/ $n$ of controls) } \\
\hline C-reactive protein (mg/dL) [median (IOR)] (102/289) & $7.75(3.36-16.6)$ & $5.90(1.8-12.1)$ & $<0.001$ \\
\hline C-reactive protein $>5$ & $66(64.7)$ & $162(56.1)$ & 0.12 \\
\hline Creatinine (mg/dL) [median (IQR)] (106/307) & $0.86(0.72-1.13)$ & $0.87(0.71-1.10)$ & 0.80 \\
\hline ASAT (UI/L) [median (IOR)] (76/253) & $29(19-43)$ & $30(22-48)$ & 0.13 \\
\hline Bilirubin (mg/dL) [median (IQR)] (70/219) & $0.7(0.5-1.0)$ & $0.5(0.3-0.7)$ & $<0.001$ \\
\hline LDH (UI/L) [median (IQR)] (66/235) & $350(253-526)$ & $276(70-1187)$ & $<0.0001$ \\
\hline LDH $>350$ & $34(51.5)$ & $60(25.6)$ & $<0.001$ \\
\hline Procalcitonin (ng/mL) [median (IQR)] (55/131) & $0.1(0.04-0.31)$ & $0.1(0.06-0.21)$ & 0.89 \\
\hline Hemoglobin (g/L) [median (IQR)] (107/308) & $13.9(12.7-15.1)$ & $13.8(6.8-17.7)$ & 0.72 \\
\hline Hemoglobin $<10$ & $2(1.9)$ & $14(4.5)$ & 0.22 \\
\hline Leucocytes count [median (IOR)] (110/308) & $9.8(7.3-12.1)$ & $6.6(4.9-9.0)$ & $<0.0001$ \\
\hline Leucocytes $>10$ & $54(49.1)$ & $56(18.2)$ & $<0.001$ \\
\hline Lymphocytes count [median (IQR)] (104/293) & $1.1(0.8-1.7)$ & $1.1(0.8-1.6)$ & 0.34 \\
\hline Platelets [median (IQR)] (108/307) & $232(183-324)$ & $206(161-258)$ & $<0.0001$ \\
\hline Platelets $<200$ & $39(36.1)$ & $144(46.9)$ & 0.05 \\
\hline APTT (s) [median (IQR)] (73/200) & $29.4(27.2-32.3)$ & $29.7(26.5-33.3)$ & 0.58 \\
\hline Prothrombine time (s) [median (IOR)] $(66 / 217)$ & $13.5(12.0-14.5)$ & $12.5(11.5-14.0)$ & 0.05 \\
\hline Fibrinogen (g/L) [median (IQR)] (53/111) & $500(386-664)$ & $500(469-614)$ & 0.61 \\
\hline D-dimer (ng/mL) [median (IOR)] (96/247) & $6021(2270-10035)$ & $680(390-1275)$ & $<0.0001$ \\
\hline D-dimer $>10000$ & $24(25.5)$ & 8 (3.3) & $<0.001$ \\
\hline D-dimer $>5000$ & $57(60.6)$ & $17(7.1)$ & $<0.001$ \\
\hline D-dimer $>3000$ & $68(72.3)$ & $26(10.8)$ & $<0.001$ \\
\hline \multicolumn{4}{|l|}{ Chest X-ray } \\
\hline Cardiomegaly & $15(16)$ & $34(10.8)$ & 0.18 \\
\hline Interstitial lung infiltrates & $51(52.6)$ & $144(73.8)$ & 0.14 \\
\hline Ground-glass lung opacities & $57(58.8)$ & $186(57.1)$ & 0.76 \\
\hline Pleural effusion & $7(7.5)$ & $14(4.4)$ & 0.22 \\
\hline \multicolumn{4}{|l|}{ Characteristics DVT in CCUS } \\
\hline Proximal & $35(40.2)$ & - & - \\
\hline Distal & 33 (37.9) & - & - \\
\hline Proximal and distal & $19(21.8)$ & - & - \\
\hline \multicolumn{4}{|l|}{ Disposition } \\
\hline Discharge from ED & $10(8.9)$ & $83(24.7)$ & $<0.001$ \\
\hline
\end{tabular}

Bold $P$ values denote statistical significance $(P<0.05)$.

APTT, activated partial thromboplastin time; ASAT, aspartate aminotransferase; CCUS, complete compression Doppler ultrasound; COVID, coronavirus disease; DVT, deep venous thrombosis; ED, emergency department; IQR, interquartile range; LDH, lactate dehydrogenase; SBP, systolic blood pressure.

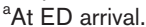

symptoms of DVT but had exceedingly high levels of D-dimer. A recent review of previous studies on VTE in COVID patients admitted to ICUs and general wards reported the presence of VTE in approximately $20 \%$ of patients with cumulative incidences of up to $35 \%$, although it did not distinguish between DVT and pulmonary embolism [18]. Two other recent studies found that the relative incidence of DVT compared to all VTE 
Table 3 Magnitude of statistically significant associations found in the unadjusted analysis

\begin{tabular}{lcc}
\hline & $\begin{array}{c}\text { Odds } \\
\text { ratio }\end{array}$ & $\begin{array}{c}95 \% \text { confidence } \\
\text { interval }\end{array}$ \\
\hline $\begin{array}{l}\text { Risk factors for developing DVT in COVID patients } \\
\quad \text { respect to COVID patients not developing DVT) }\end{array}$ & & \\
D-dimers $>5000 \mathrm{ng} / \mathrm{mL}$ & 19.44 & $10.53-35.88$ \\
Leucocyte count $>10 \mathrm{cells} / \mu \mathrm{L}$ & 4.65 & $2.91-7.43$ \\
Surgery/immobilization $<1$ month & 3.28 & $1.51-7.12$ \\
History of VTE & 2.98 & $1.31-6.74$ \\
Lactate dehydrogenase $>350 \mathrm{UI} / \mathrm{L}$ & 2.00 & $1.22-3.27$ \\
Room air pulsioxymetry at ED arrival $<96 \%$ & 1.99 & $1.23-3.21$ \\
Complaining with chest pain & 1.80 & $1.03-3.17$ \\
Hypertension & 1.73 & $1.12-2.67$ \\
Age $>65$ & 1.50 & $1.02-2.45$ \\
Complaining of fever & 0.48 & $0.31-0.75$ \\
Complaining of cough & 0.40 & $0.26-0.63$ \\
Complaining of rhinorrhea & 0.26 & $0.05-1.06$ \\
\hline
\end{tabular}

COVID, coronavirus disease; DVT, deep venous thrombosis; ED, emergency department; VTE, venous thromboembolism.

events in COVID patients ranged from 1.4 [13] to $13 \%$ [9] of all VTE episodes, being percentages which very much lower than those reported in the general population (non-COVID patients), in which DVT is present in up to $53 \%$ of VTE episodes [19]. Taking all these data together with our results, we believe that COVID patients do not have an increased risk of presenting this type of VTE. In summary, the incidence of confirmed DVT in patients with COVID who attend the ED, in the same way as pulmonary embolism [20], does not seem to be increased.

The clinical characteristics of COVID patients that were found to be associated with a higher risk of presenting DVT were older age and having a previous history of VTE, surgery or immobilization during the last month, similar to what is observed in the general population [21]. Interestingly, COVID patients in the ED presented fewer symptoms related to the infectious process such as fever, cough but more frequently had analytical inflammatory parameters such as increased white blood cell count and LDH values. Similar data have been reported in hospitalized patients with COVID and DVT and suggest a multifactorial cause or association with advanced age, infectious processes, and inflammatory and coagulopathic state [22]. Significantly, we found D-dimer levels above 5000 to be strongly associated with the presence of DVT in COVID patients. The association of disease severity and mortality in COVID-19 with higher D-dimer levels has been reported in several studies [23], but it is a nonspecific marker that can rise in many conditions, including VTE. In this sense, several retrospective publications found a relationship between higher D-dimer levels and VTE (both DVT and pulmonary embolism) in COVID-19 patients. For this reason, some authors suggest the need for a targeted search for DVT or pulmonary embolism if D-dimer levels rise above $2000-4000 \mu \mathrm{g} / \mathrm{L}$ [24]. However, recent guidelines do not suggest the use of biomarkers in the diagnostic evaluation for suspected DVT or pulmonary embolism in in-patient populations
[25]. In this sense, our findings are significant because they have been obtained in the context of outpatients assessed in the ED.

Nearly $40 \%$ of DVTs are asymptomatic in COVID patients. This can be explained in that most hospital protocols created in Spain at the beginning of the outbreak indicated the search for DVT or if the D-dimer levels were high. Furthermore, this incidence is higher than that observed in other studies. In a prospective study in patients admitted to a general ward with COVID-19 pneumonia and elevated D-dimer levels, the incidence of asymptomatic DVT was similar to that described in other non-COVID series, being around 15\% [11,26]. Remarkably, we found less frequency for simultaneous proximal and distal DVT in patients with COVID-19. This fact, combined with the finding of a lower pulmonary embolism thrombus load in COVID patients, may indicate a different pathophysiological process, which would be more related to a procoagulant state than to a thromboembolic mechanism. Similar results have recently been reported in a larger study with pulmonary embolism patients, suggesting a different phenotype of thrombotic disease $[27,28]$.

Nearly all the outcomes assessed in UMC-19-S were worse for COVID patients with DVT compared to the control group, even after adjustment for age and sex. But in-hospital mortality was similar. The presence of a DVT may require the need for hospitalization and prolong the hospital stay, probably related to anticoagulation control. Furthermore, these patients probably have a more severe COVID (desaturation, increased LDH and D-dimer) in addition to greater comorbidity such as older age. The absence of differences in mortality could indicate that the presence of DVT itself in COVID patients is not a risk factor for mortality.

\section{Limitations}

This study has several limitations. First, the relative frequency of DVT implies both symptomatic DVT and asymptomatic DVT, and thus, comparisons with epidemiological studies may not be valid. Second, in many cases, the diagnosis of COVID-19 was based on clinical and radiological findings with no microbiological confirmation. However, the sensitivity analysis including only COVID patients microbiologically confirmed by RT-PCR did not found differences in outcomes. Third, we did not adjust our estimates for all relevant patient-related or disease-related factors, especially pulmonary embolism, and this could somewhat alter the estimations presented in the current study. This could also be true for the estimations of outcomes, because the limited number of cases included in our study and adverse events observed were only adjusted for age and sex. Fifth, although the case record form was standardized, as a retrospective study, there was no monitoring of data collection methods. 


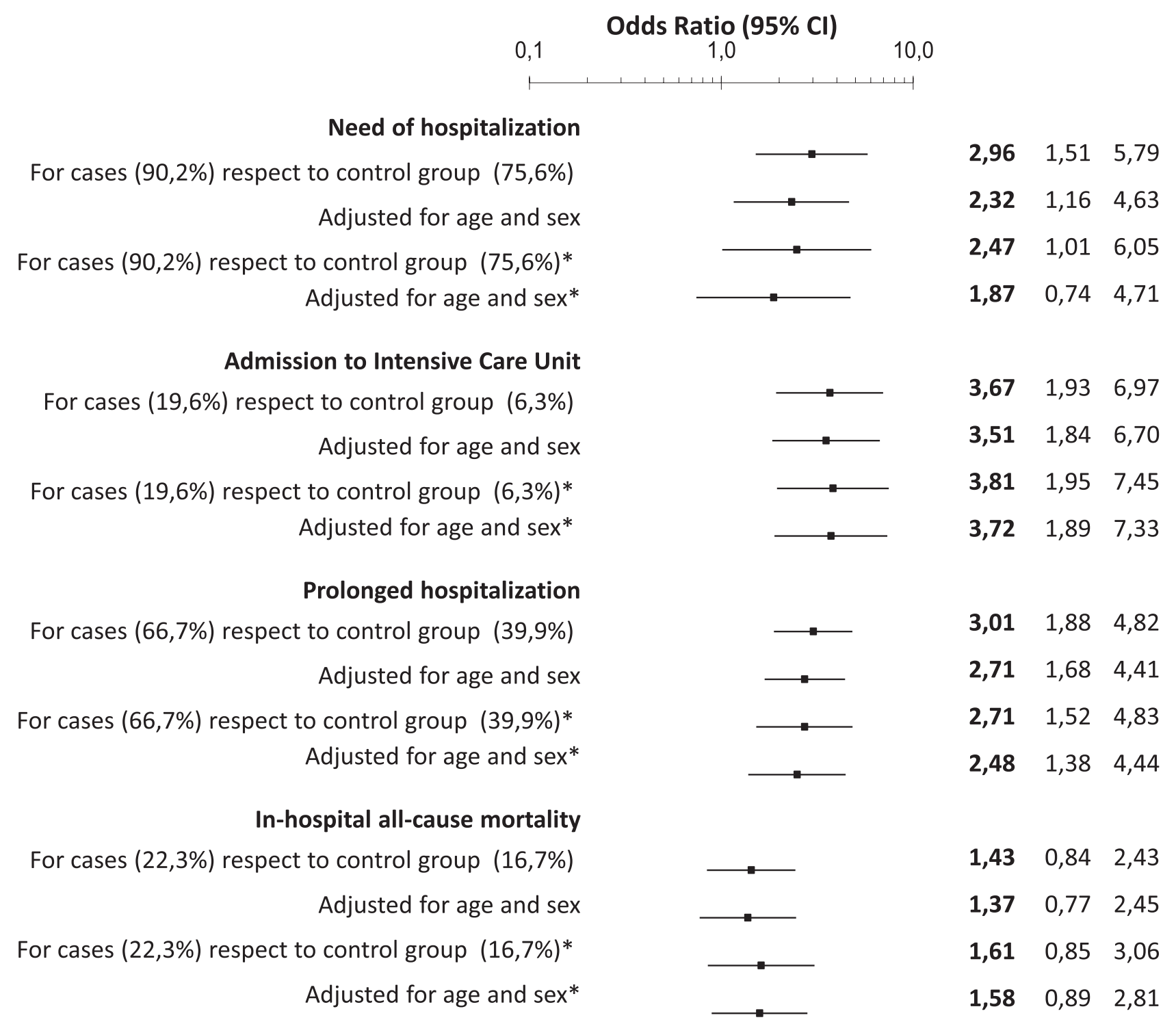

Outcomes of patients with COVID-19 and deep venous thrombosis (DVT) (cases) compared with patients with COVID-19 without DVT (control group), unadjusted and adjusted for age and sex. ${ }^{*}$ Results of the sensitivity analysis including only COVID patients microbiologically confirmed by RT-PCR ( 80 cases and 246 controls).

\section{Conclusion}

Despite these limitations, we conclude that the incidence of DVT in COVID patients attending the ED is similar than that observed in non-COVID patients. In some circumstances, this incidence could be increased, especially in COVID patients with a history of VTE, recent surgery/ immobilization and D-dimer levels above $5000 \mathrm{ng} / \mathrm{mL}$. Patients with COVID developing DVT have poor prognosis but in-hospital mortality is similar to that observed in COVID patients without DVT.

\section{Acknowledgements}

All authors discussed the idea and design of study and provided patients. Data analysis and first draft writing was done by O.M. All authors read the draft and provided insight for the final version. O.M. is the guarantor of the article, taking responsibility for the integrity of the work, from inception to publication. The present work was performed without any direct or indirect financial support. Steering Committee: Ò.M. and S.J. (Hospital Clínic, Barcelona), J.G.del.C., F.J.M-S. and E.J.G-L. (Hospital Clínico San Carlos, Madrid), P.L. (Hospital General de Alicante), G.B-P. (Hospital Universitario de Canarias, Tenerife), A.M. (Hospital Universitario Severo Ochoa de Leganés, Madrid), P.P.S. (Hospital General Universitario Reina Sofía, Murcia), A.A-A. (Hospital de la Santa Creu i Sant Pau), J.J. (Hospital Universitari de Bellvitge, Barcelona). 


\section{Conflicts of interest}

There are no conflicts of interest.

\section{References}

1 Guan WJ, Ni ZY, Hu Y, Liang WH, Ou CQ, He JX, et al.; China Medical Treatment Expert Group for Covid-19. Clinical characteristics of Coronavirus Disease 2019 in China. N Engl J Med 2020; 382:17081720.

2 Chen N, Zhou M, Dong X, Qu J, Gong F, Han Y, et al. Epidemiological and clinical characteristics of 99 cases of 2019 novel coronavirus pneumonia in Wuhan, China: a descriptive study. Lancet 2020; 395:507-513.

3 Richardson S, Hirsch JS, Narasimhan M, Crawford JM, McGinn T, Davidson KW, et al.; the Northwell COVID-19 Research Consortium. Presenting characteristics, comorbidities, and outcomes among 5700 patients hospitalized with COVID-19 in the New York City area. JAMA 2020; 323:2052-2059.

4 Lodigiani C, lapichino G, Carenzo L, Cecconi M, Ferrazzi P, Sebastian T, et al.; Humanitas COVID-19 Task Force. Venous and arterial thromboembolic complications in COVID-19 patients admitted to an academic hospital in Milan, Italy. Thromb Res 2020; 191:9-14.

5 Zhou F, Yu T, Du R, Fan D, Liu Y, Liu Z, et al. Clinical course, and risk factors for mortality of adult in-patients with COVID-19 in Wuhan, China: a retrospective cohort study [published correction appears in Lancet. 2020 Mar 28:395(10229):1038] [published correction appears in Lancet. 2020 Mar 28;395(10229):1038]. Lancet 2020; 395:1054-1062.

6 Yao W, Wang T, Jiang B, Gao F, Wang L, Zheng H, et al.; collaborators. Emergency tracheal intubation in 202 patients with COVID-19 in Wuhan, China: lessons learnt and international expert recommendations. $\mathrm{Br} J$ Anaesth 2020; 125:e28-e37.

7 Danzi GB, Loffi M, Galeazzi G, Gherbesi E. Acute pulmonary embolism and COVID-19 pneumonia: a random association? Eur Heart J 2020; 41: 1858.

8 Cui S, Chen S, Li X, Liu S, Wang F. Prevalence of venous thromboembolism in patients with severe novel coronavirus pneumonia. J Thromb Haemost 2020; 18:1421-1424.

9 Middeldorp S, Coppens M, van Haaps TF, Foppen M, Vlaar AP, Müller $\mathrm{MCA}$, et al. Incidence of venous thromboembolism in hospitalized patients with COVID-19. J ThrombHaemos 2020; 18:1995-2002.

10 Klok FA, Kruip MJHA, van der Meer NJM, Arbous MS, Gommers D, Kant $\mathrm{KM}$, et al. Confirmation of the high cumulative incidence of thrombotic complications in critically ill ICU patients with COVID-19: an updated analysis. Thromb Res 2020; 191:148-150.

11 Helms J, Tacquard C, Severac F, Leonard-Lorant I, Ohana M, Delabranche X, et al.; CRICS TRIGGERSEP Group (Clinical Research in Intensive Care and Sepsis Trial Group for Global Evaluation and Research in Sepsis). High risk of thrombosis in patients with severe SARS-CoV-2 infection: a multicenter prospective cohort study. Intensive Care Med 2020; 46:1089-1098.

12 Poissy J, Goutay J, Caplan M, Parmentier E, Duburcq T, Lassalle F, et al.; Lille ICU Haemostasis COVID-19 Group. Pulmonary embolism in patients with COVID-19: awareness of an increased prevalence. Circulation 2020 142:184-186.

13 Demelo-Rodríguez P, Cervilla-Muñoz E, Ordieres-Ortega L, Parra-Virto A, Toledano-Macías M, Toledo-Samaniego N, et al. Incidence of asymptomatic deep vein thrombosis in patients with COVID-19 pneumonia and elevated D-dimer levels. Thromb Res 2020; 192:23-26.
14 Ministry of Health.Spanish Government. https://www.mscbs.gob.es/. 2020 https://cnecovid.isciii.es/covid19/\#niveles-de-gravedad. [Accessed 1 June 2020].

15 Gil-Rodrigo A, Miró O, Piñera P, Burillo-Putze G, Jiménez S, Martín A, et al. Analysis of clinical characteristics and outcomes in patients with COVID19 based on a series of 1000 patients treated in Spanish emergency departments. Emergencias. 2020; 32:233-41

16 Miró O, González Del Castillo J. Collaboration among Spanish emergency departments to promote research: on the creation of the SIESTA (Spanish Investigators in Emergency Situations TeAm) network and the coordination of the UMC-19 (Unusual Manifestations of COVID-19) macroproject. Emergencias 2020; 32:269-77.

17 Pollán M, Pérez-Gómez B, Pastor-Barriuso R, Oteo J, Hernán MA, PérezOlmeda M, et al.; ENE-COVID Study Group. Prevalence of SARS-CoV-2 in Spain (ENE-COVID): a nationwide, population-based seroepidemiological study. Lancet 2020; 396:535-544.

18 Al-Ani F, Chehade S, Lazo-Langner A. Thrombosis risk associated with COVID-19 infection. A scoping review. Thromb Res 2020; 192:152-160.

19 Maestre A, Sánchez R, Rosa V, Aujesky D, Lorenzo A, Barillari G, Monreal $\mathrm{M}$; RIETE Investigators. Clinical characteristics and outcome of inpatients versus outpatients with venous thromboembolism: findings from the RIETE Registry. Eur J Intern Med 2010; 21:377-382.

20 Freund Y, Drogrey M, Miró Ò, Marra A, Féral-Pierssens AL, Penaloza A, et al.; IMPROVING EMERGENCY CARE FHU Collaborators. Association between pulmonary embolism and COVID-19 in emergency department patients undergoing computed tomography pulmonary angiogram: the PEPCOV international retrospective study. Acad Emerg Med 2020; 27:811-820.

21 Heit JA, Spencer FA, White RH. The epidemiology of venous thromboembolism. J Thromb Thrombolysis 2016; 41:3-14.

22 Zhang L, Feng X, Zhang D, Jiang C, Mei H, Wang J, et al. Deep vein thrombosis in hospitalized patients with Coronavirus disease 2019 (COVID-19) in Wuhan, China: prevalence, risk factors, and outcome. Circulation 2020; 142:114-128.

23 Tang N, Li D, Wang X, Sun Z. Abnormal coagulation parameters are associated with poor prognosis in patients with novel coronavirus pneumonia. J Thromb Haemost 2020; 18:844-847.

24 Oudkerk M, Büller HR, Kuijpers D, van Es N, Oudkerk SF, McLoud T, et al. Diagnosis, prevention, and treatment of thromboembolic complications in COVID-19: report of the National Institute for Public Health of the Netherlands. Radiology 2020; 297:E216-E222.

25 Moores LK, Tritschler T, Brosnahan S, Carrier M, Collen JF, Doerschug K, et al. Prevention, diagnosis, and treatment of venous thromboembolism in patients with COVID-19: CHEST guideline and expert panel report. Chest 2020; 297:1143-1163.

26 Ciuti G, Grifoni E, Pavellini A, Righi D, Livi R, Perfetto F, et al. Incidence and characteristics of asymptomatic distal deep vein thrombosis unexpectedly found at admission in an Internal Medicine setting. Thromb Res 2012; 130:591-595.

27 van Dam LF, Kroft LJM, van der Wal LI, Cannegieter SC, Eikenboom J, de Jonge $\mathrm{E}$, et al. Clinical and computed tomography characteristics of COVID-19 associated acute pulmonary embolism: A different phenotype of thrombotic disease? Thromb Res 2020; 193:86-89.

28 Cattaneo M, Bertinato EM, Birocchi S, Brizio C, Malavolta D, Manzoni M, et al. Pulmonary embolism or pulmonary thrombosis in COVID-19? Is the recommendation to use high-dose heparin for thromboprophylaxis justified? Thromb Haemost 2020; 120:1230-1232. 